\title{
Multiscale Investigation of Porosity Characteristic in Concrete Exposed to Acidic Environment
}

\author{
Y. F. Fan, H. Y. Luan, and S. Y. Zhang \\ Institute of Road and Bridge Engineering, Dalian Maritime University, Dalian, Liaoning 116026, China \\ Correspondence should be addressed to Y. F. Fan, fanyf72@yahoo.com.cn
}

Received 24 February 2012; Accepted 16 May 2012

Academic Editor: Citlalli Gaona Tiburcio

Copyright () 2012 Y. F. Fan et al. This is an open access article distributed under the Creative Commons Attribution License, which permits unrestricted use, distribution, and reproduction in any medium, provided the original work is properly cited.

Porosity has a significant effect on the mechanical response of concrete. It is essential to well understand the relation between porosity characteristics inside the concrete with the damage process of concrete exposed to the aggressive environment. To simulate the acidic environment, the acid solutions with $\mathrm{pH}$ level of 1.5 and 2.5 were deposed by the mixture of the sulfate and nitric acid in the laboratory. Computed tomography (CT) and scanning electron microscopy (SEM) were used to characterize the microstructures of concrete exposed to the acidic solution for the scheduled periods. Pore distribution and porosity ratio of concrete specimens suffering various damage processes are obtained. The CT digital images were analyzed by Pro-Plus software. The threshold value is suggested to identify the pore. The developments of pore structure and porosity ratio of the concrete samples are examined. The relation between the porosity characteristic and mechanical response of concrete is discussed.

\section{Introduction}

The porosity characteristic inside the concrete greatly influences its permeability, mechanical response, and durability. The intensity of the interactions with the aggressive agents and external loads always lead to the change of the pore structure inside concrete [1]. To well understand the damage process of concrete, it is essential to discover the porosity characteristics inside the concrete under different damage states.

Water vapor adsorption and nitrogen adsorption test and mercury intrusion porosimetry (MIP) test are the most popular methods used to examine the porosity characteristics inside concrete [2, 3]. However, MIP results are affected by many factors and depend heavily on the experimental procedure adopted [3, 4]. Ultrasonics, as a kind of nondestructive technique, has been used to assess porosity inside concrete [5]. But the research reports that it is quite difficult to obtain precise evaluations of the ultrasonic parameters.

In this study, the pore structures of concrete exposed to aggressive environment were investigated from multiscale levels. The acid solutions with $\mathrm{pH}$ level of 1.5 and 2.5 deposed by the mixture, the sulfate and nitric acid in the laboratory were considered. After being exposed to the acidic solution for the scheduled periods, the concrete samples were picked out. The removed specimens were first dried for about two to three days, followed by the physical, CT, and SEM/EDS tests. Pore distribution and porosity ratio of concrete specimens suffering various damage processes are obtained. The CT digital images were analyzed by Pro-Plus software. The threshold value is suggested for the identification of the pore. The developments of pore structure and porosity ratio inside the concrete samples are examined. The relation between the porosity characteristic and physical response of concrete are discussed.

\section{Experimental Programme}

2.1. Specimen Preparation. Commercial concrete were used in this study. Cubic samples with the dimension of $150 \times$ $150 \times 150 \mathrm{~mm}^{3}$ were prepared. Mix proportion of the concrete mixtures is shown in Table 1.

2.2. Accelerated Corrosion Procedure. Acidic solution with $\mathrm{pH}$ level of 1.5 and 2.5 were deposed by the mixture of sulfate and nitric acid solutions (molar ratio is 9:1) in the laboratory, 
TABLE 1: Mix proportion of the concrete mixtures.

\begin{tabular}{lccccccc}
\hline w/c & w $/ \mathrm{b}$ & Cement $/ \mathrm{kg} / \mathrm{m}^{3}$ & Sand $/ \mathrm{kg} / \mathrm{m}^{3}$ & Coarse aggregate $/ \mathrm{kg} / \mathrm{m}^{3}$ & Water $/ \mathrm{kg} / \mathrm{m}^{3}$ & Fly ash $/ \mathrm{kg}$ & Reducing-water agent $/ \mathrm{kg}$ \\
\hline 0.353 & 0.304 & 450 & 678 & 1040 & 159 & 60.0 & 12.8 \\
\hline
\end{tabular}

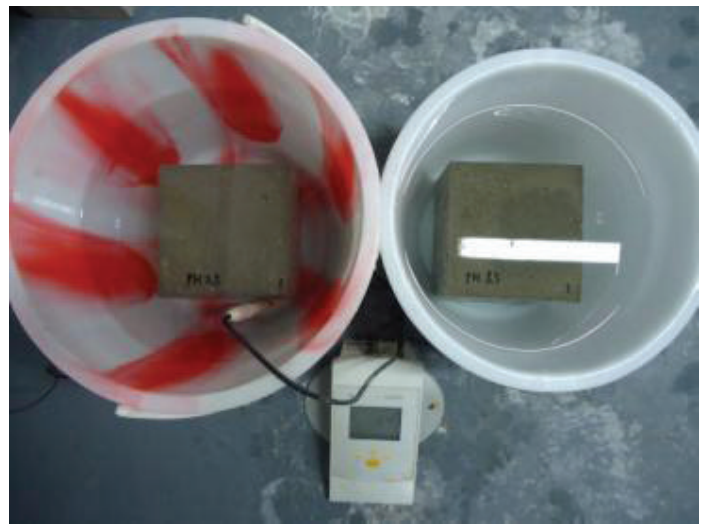

Figure 1: Concrete specimens conditioned in the mixed acid solution.

TABLE 2: Condition details.

\begin{tabular}{lccc}
\hline Designation & Exposing condition & $\begin{array}{c}\text { Solution } \\
\text { acidity }\end{array}$ & $\begin{array}{c}\text { Immersion } \\
\text { time (days) }\end{array}$ \\
\hline Series $\mathrm{C} 1$ & $\begin{array}{c}\mathrm{H}_{2} \mathrm{SO}_{4}+\mathrm{HNO}_{3} \\
(\text { molar ratio is 9: } 1)\end{array}$ & $\mathrm{pH} 1.5$ & $0,5,10,15,20$ \\
\hline Series $\mathrm{C} 2$ & $\begin{array}{c}\mathrm{H}_{2} \mathrm{SO}_{4}+\mathrm{HNO}_{3} \\
(\text { molar ratio is 9: } 1)\end{array}$ & $\mathrm{pH} 2.5$ & $0,5,10,15,20$ \\
\hline
\end{tabular}

respectively. A plastic tank was used to contain acid solution and the concrete specimens. After curing for 28 days, the concrete specimens were divided into two groups. One batch of specimens was continuously cured in water with $\mathrm{pH}$ level of 1.5 and was denoted as series $C 1$. The other batch was immersed in the tank filled with acid solution with $\mathrm{pH}$ level of 2.5 and was denoted as series C2. Exposure protocols were listed in detail in Table 2. Acidity of the solution was recorded by $\mathrm{PB}-10$ sartorius acidometer. The $\mathrm{pH}$ value of the acid solution was detected by a digital $\mathrm{pH}$ meter [6]. To keep the $\mathrm{pH}$ level of the mixed solution constant, nitric acid solution was added periodically. At the same time, the solution was stirred thoroughly to reduce differential concentrations of the acid inside the solution tank (shown in Figure 1).

After being exposed to the acidic solution for the scheduled periods listed in Table 2, the samples were picked out. The removed specimens were first dried for about two to three days, followed by the physical, CT, and SEM/EDS tests.

\subsection{Methods to Determine Porosity inside the Specimen}

2.3.1. Scanning Electron Microscopy (SEM). SEM is a wellestablished method that can offer useful information concerning the structural of material [7]. To better understand the evolution of microstructure of the concrete samples exposed to acidic solution with certain periods ( 0 day, 3 days,
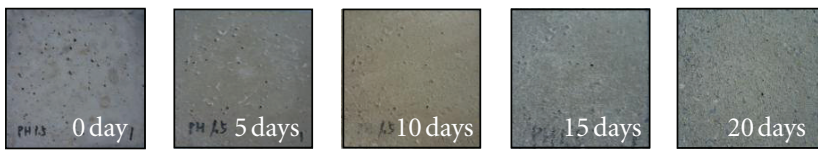

(a) $\mathrm{pH} 1.5$
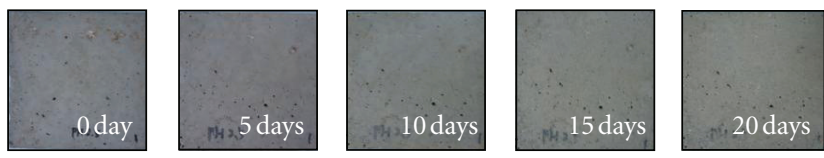

(b) $\mathrm{pH} 2.5$

FIGURE 2: The change of surface appearance on concrete specimens exposed to acidic solution.

7 days, and 10 days), microstructures of the samples were observed by JSM-6360LV SEM system. Energy dispersive spectroscopy (EDS) technique was applied to analyze the elemental distribution in the samples as well. The EDS plots provide the presence of the chemical elements, which will confirm the composition of crystal in the concrete samples.

2.3.2. Computer Tomography (CT). Since the X-rays are absorbed by the concrete specimen according to composition and density of the material, different features can be detected. Objects with a higher density absorb more X-rays, resulting in bright areas. Consequently, the lower the density, the less $\mathrm{X}$-rays are absorbed, creating darker regions. That means, the pores appear very dark. Accordingly, the pores can be distinguished and quantified by image analysis of the $2 \mathrm{D}$ sections. In this research, CT technology was selected to describe the porosity character of concrete specimen under different damage states.

For the measurements, a "Siemens somatom sensation" 16-slice spiral computed tomography scanner made in Germany was used. Samples were scanned with a fixed X-ray source, at $140 \mathrm{kV}, 200 \mathrm{~mA}$, and $22.60 \mathrm{mGy}$ CTD. Each scan was $1 \mathrm{~mm}$ thick and the total number of the scans was about 200.

\section{Results and Discussions}

3.1. Visual Observation. From visual inspection of the samples exposure to acid solution for various periods (shown in Figure 2), it can be seen that the surface become to be yellow, and the surface dissolution of cement paste lead to exposure of aggregates.

3.2. Mass Change. Based on the visual observations described above, it was obvious that honeycomb voids were formed as conditioning continued, causing the mass change. This could be measured by an electronic scale with an 


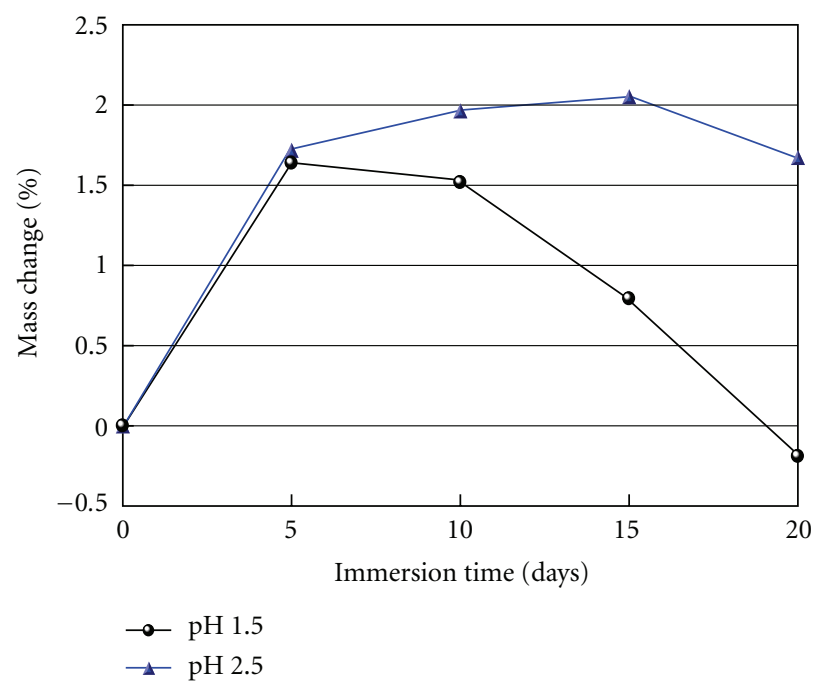

(a) Relation between the mass change and conditioning time

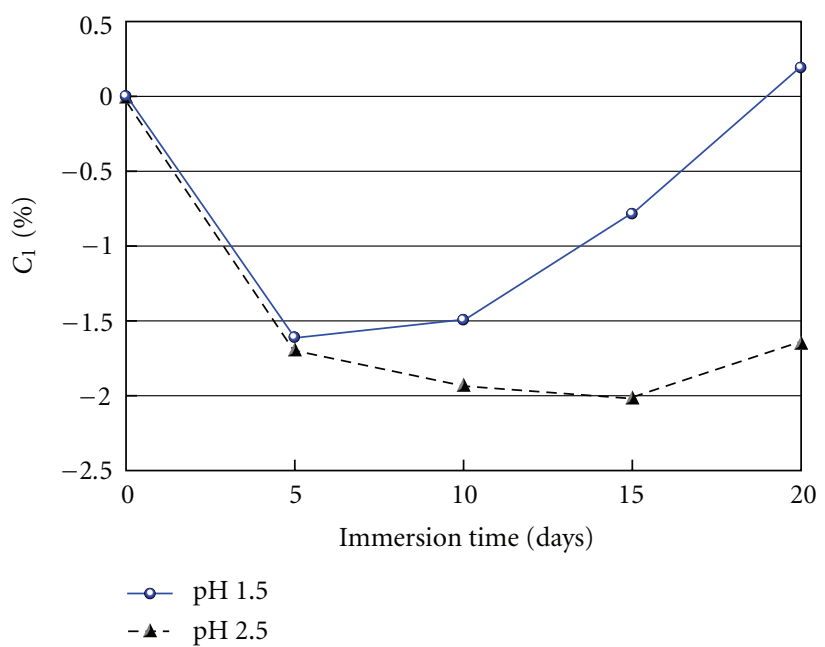

(b) Relation between $C_{1}$ and conditioning time

FIGURE 3: Relation between void volume and immersion time.

accuracy of $0.1 \mathrm{~g}$. Relation between the mass change and conditioning time is given in Figure $3(\mathrm{a})$. Based on the theory of mesomechanics, concrete can be considered as a combined material which consisted of base material and void. Relation between the volume of base material and voids can be expressed as

$$
\begin{aligned}
& C_{1}=\frac{m_{t 0}-m_{t t}}{m_{t t}}, \\
& C_{1}+C_{0}=1,
\end{aligned}
$$

where, $m_{t t}$ is the mass of concrete specimens immersed in the acid solution for $t$ days; $m_{t 0}$ is the mass of undamaged concrete specimens; $C_{1}$ is the volume of voids; $C_{0}$ is the volume of base material. In this way, the relation between the developments of voids volume over time can be obtained quantitatively, which is shown in Figure 3(b).

3.3. Scanning Electron Microscopy (SEM). SEM micrographs of concrete samples at different exposure times are achieved, which are shown in Figure 4. The EDS spectra of concrete samples exposed to the acid solution is shown in Figure 4 as well.

From the SEM images, it is obvious that progressive and significant alternation occurs in the internal microstructures of concrete samples during the exposure periods. With the development of exposure time, the internal pore diameter become wider, and the microcracks in the samples become wider and longer. What is more, the morphology of internal microstructure in the samples becomes softening with the prolongation of the exposure time.

Owing to the chemical reactions between the acid solution and concrete, the compositions in the concrete are changed. To clarify the composition of products generated by acid attack, EDS were carried out on the surface of products shown in SEM micrograph. EDS plots show the presence of calcium, silicon, aluminum, oxygen, magnesium, ferrum, and kalium. The principal elements are calcium, silicon, aluminum, and oxygen. From EDS test, an indication of the number of atoms in the species being examined is obtained as well. The number of elemental and atomics distribution in concrete samples at various exposure times are counted and percentages are ascribed, respectively, which are plotted in Figure 5.

From Figure 5, it is clear that the content of calcium has a significant reduction and the content of silicon has an obvious increase in the samples exposed to acid solution. This result is in good agreement with the interpretation of deteriorating mechanism of concrete in acid medium reported by other researches [8-10]. That is, more soluble products are formed through the chemical reaction between and nitric acid, sulfate acid and calcium hydroxide. Therefore, calcium compounds of cement paste formed in concrete through the hydration process and the calcium in calcareous aggregate are leached away, and silica gel or sometimes magnesium silicate hydrate fill the space.

\subsection{CT Digital Image Analysis}

3.4.1. Digital Image. After curing the concrete prism specimen in water for 28 days, it was taken out and scanned. Each scan was $1 \mathrm{~mm}$ thick, and the total number of the scans was about 150. The internal mesolevel structure of the specimen is specified based on the 150 scanning images. Then, the specimens were conditioned in the simulated acid solution for further corrosion and scanned again at the scheduled time.

The output scanning section of concrete is recorded by the document of DCM format, and the CT number is stored in 12 digital capacities. Therefore, each pixel can display 4096 degrees. The output digital image is 512 pixel points $\times 512$ pixel points (shown in Figure 6). 

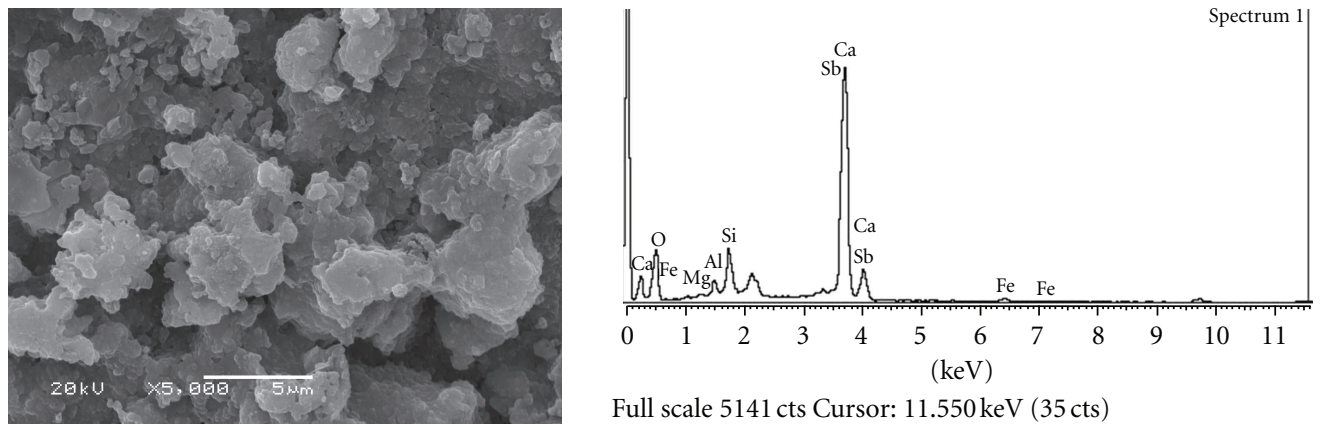

Full scale 5141 cts Cursor: $11.550 \mathrm{keV}$ ( 35 cts)

(a)
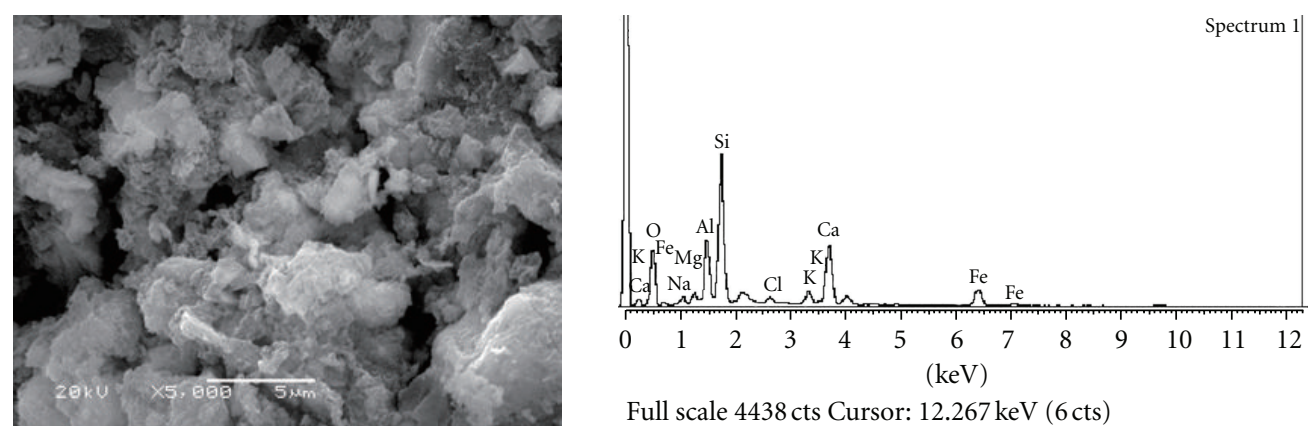

(b)
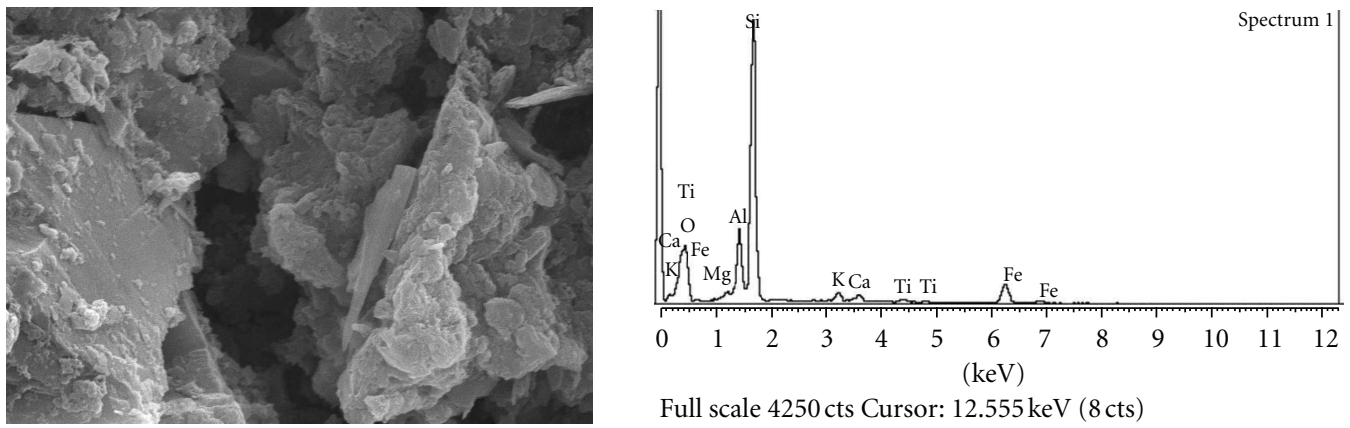

Full scale 4250 cts Cursor: $12.555 \mathrm{keV}$ ( $8 \mathrm{cts})$

(c)
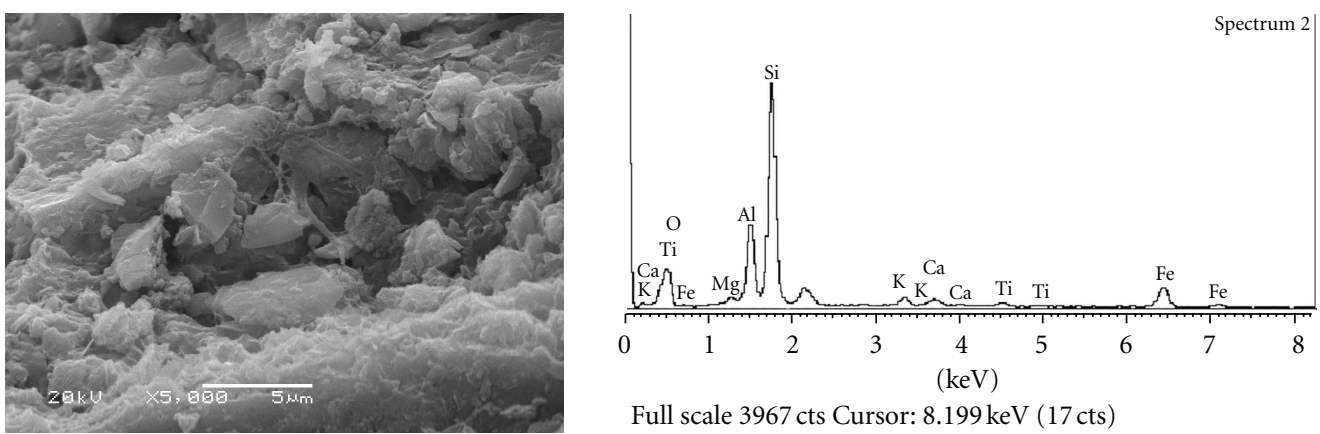

Full scale 3967 cts Cursor: $8.199 \mathrm{keV}$ (17 cts)

(d)

FIGURE 4: SEM micrograph $(\times 5000)$ and EDS spectra for concrete attacked by acid ((a) controlled specimen; (b) specimen exposed to acid for 3 days; (c) specimen exposed to acid for 7 days; (d) specimen exposed to acid for 10 days).

3.4.2. Threshold Value for Identifying the Pore Structure. To identify the distribution of pores inside the concrete, binary processing was executed on the scanning digital images. According to the theory of gray threshold method, a threshold value should be determined firstly. Then, the gray scale values of each pixel are compared with the threshold value. If the gray scale value on the pixel point is bigger than 255 , the gray scale value on this pixel point is 255 , otherwise it is zero. Therefore, the primary problem is to determine a reasonable threshold value. In this research, 


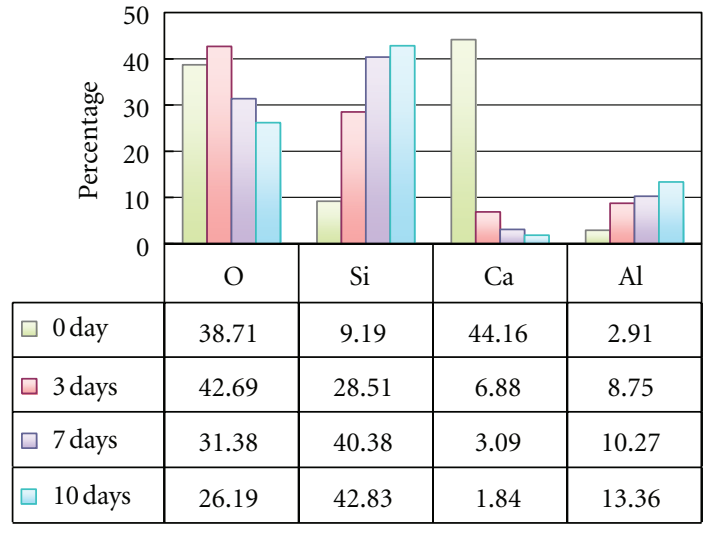

(a) normalized weight content (unit: \%)

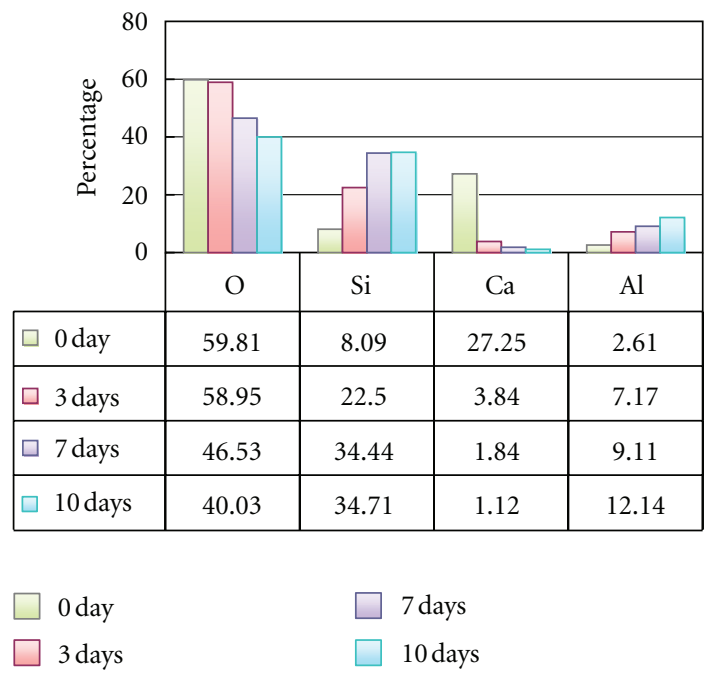

(b) atomic content(unit: \%)

Figure 5: Compositional analysis of EDS spectra for concrete samples.
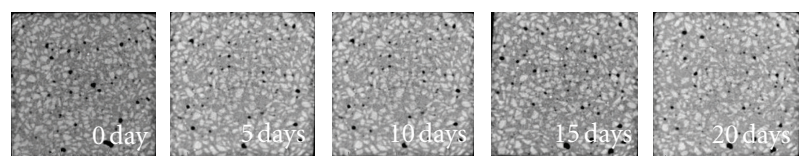

FIGURE 6: CT scanning images of concrete specimen conditioning in acidic solution with $\mathrm{pH}$ level of 1.5 .

image segmentation technology was applied for determining the threshold value. Gray histogram of the scanning images was obtained by Matlab software, which is given in Figure 7.

From Figure 7, it can be seen that the image have double peaks. The objective and background can be divided into two parts by the gray scale value at the valley bottom. It can be derived that the range of the threshold value is between 70 and 82 . The pore inside concrete was recognized by the software of Pro-Plus. The obtained pore-recognition graph for one section is plotted in Figure 8. Assuming threshold value is from 70 to 82 , porosity ratio for each section can be calculated (Figure 9).

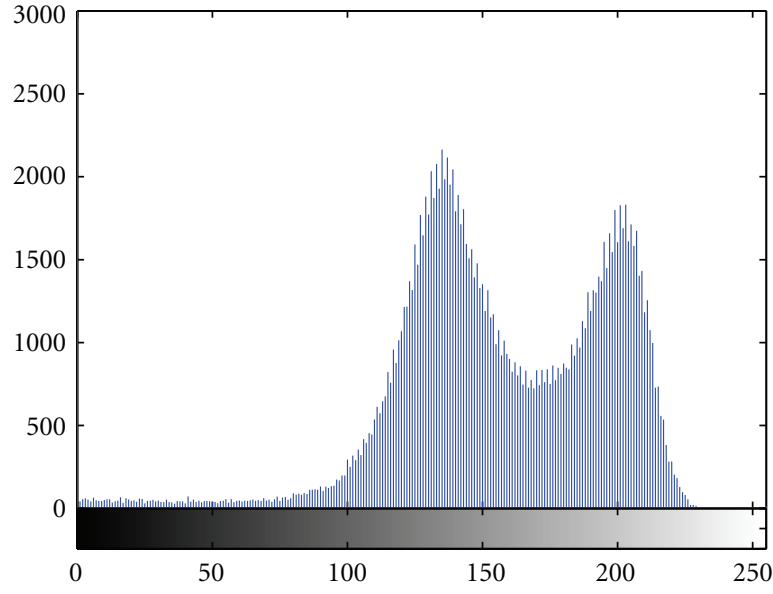

FIGURE 7: Gray histogram of the scanning images.

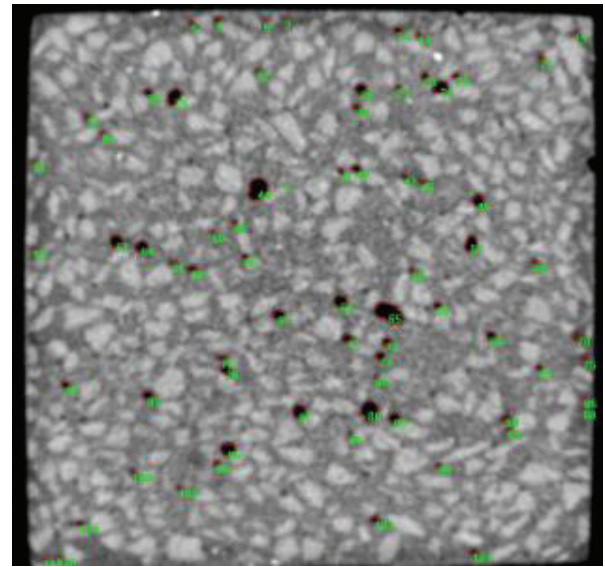

FIGURE 8: Pore recognition graph.

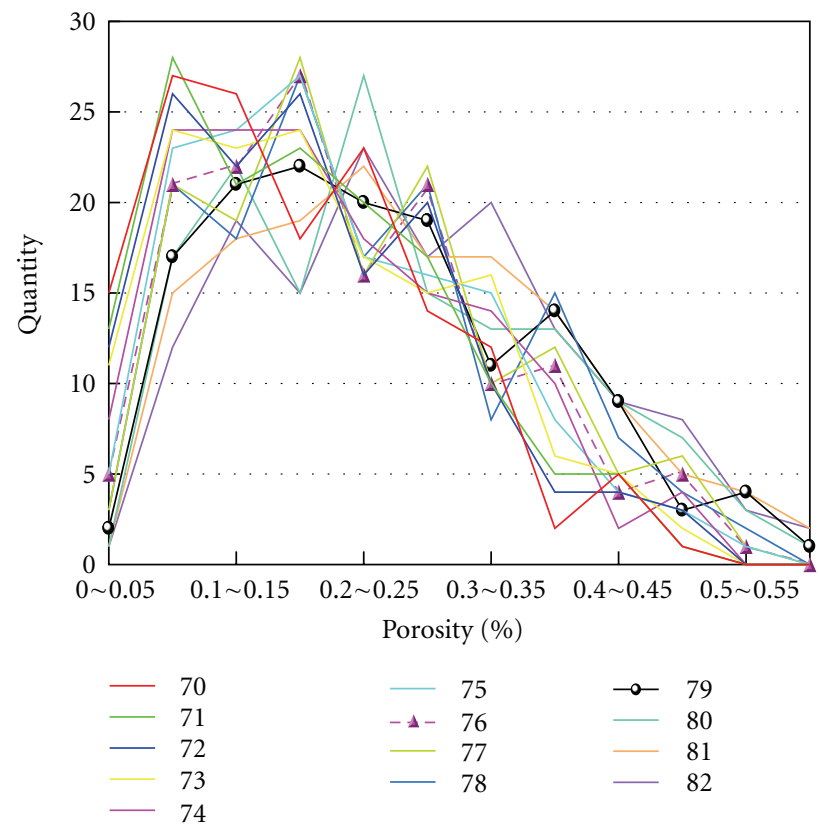

FIGURE 9: Distribution of porosity ratio. 


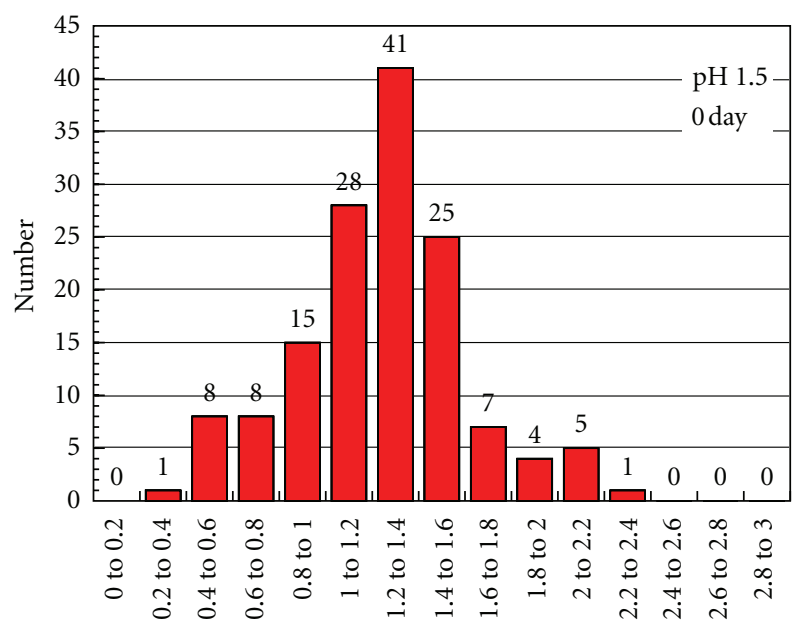

Porosity (\%)

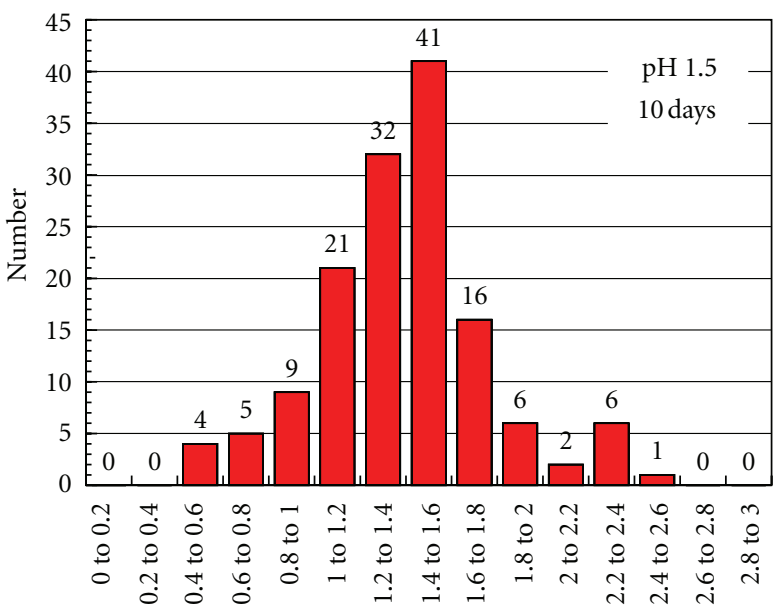

Porosity (\%)

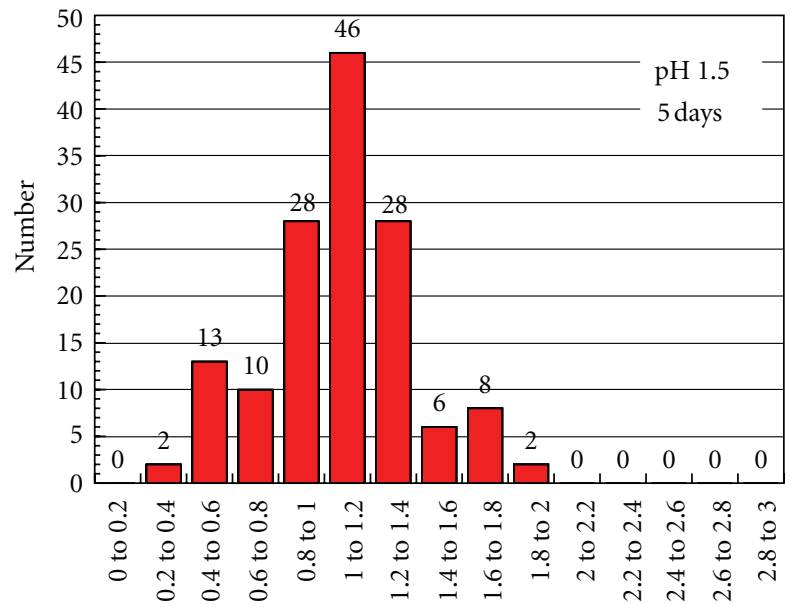

Porosity (\%)

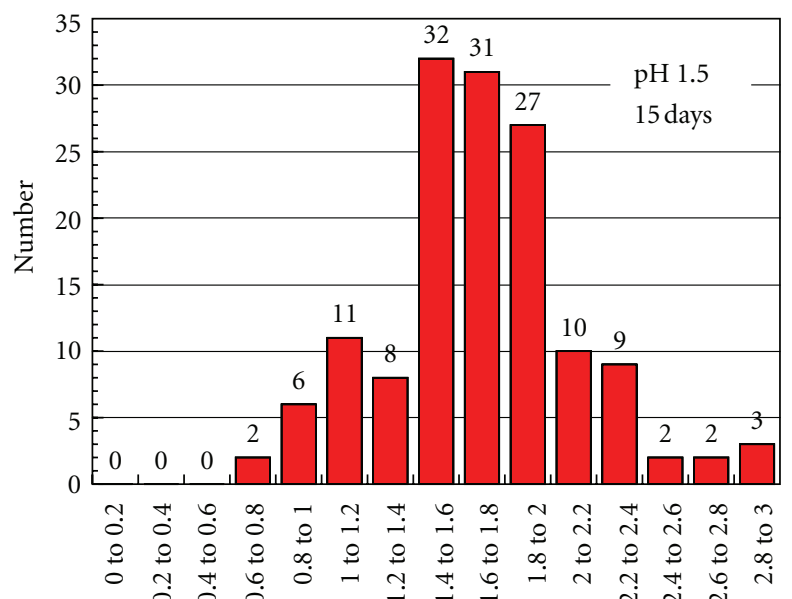

Porosity (\%)

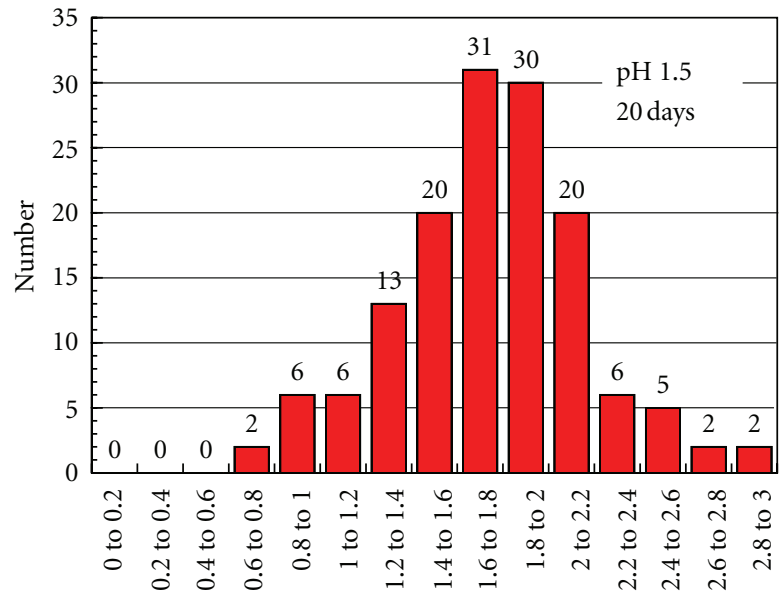

Porosity (\%)

(a)

FIgure 10: Continued. 

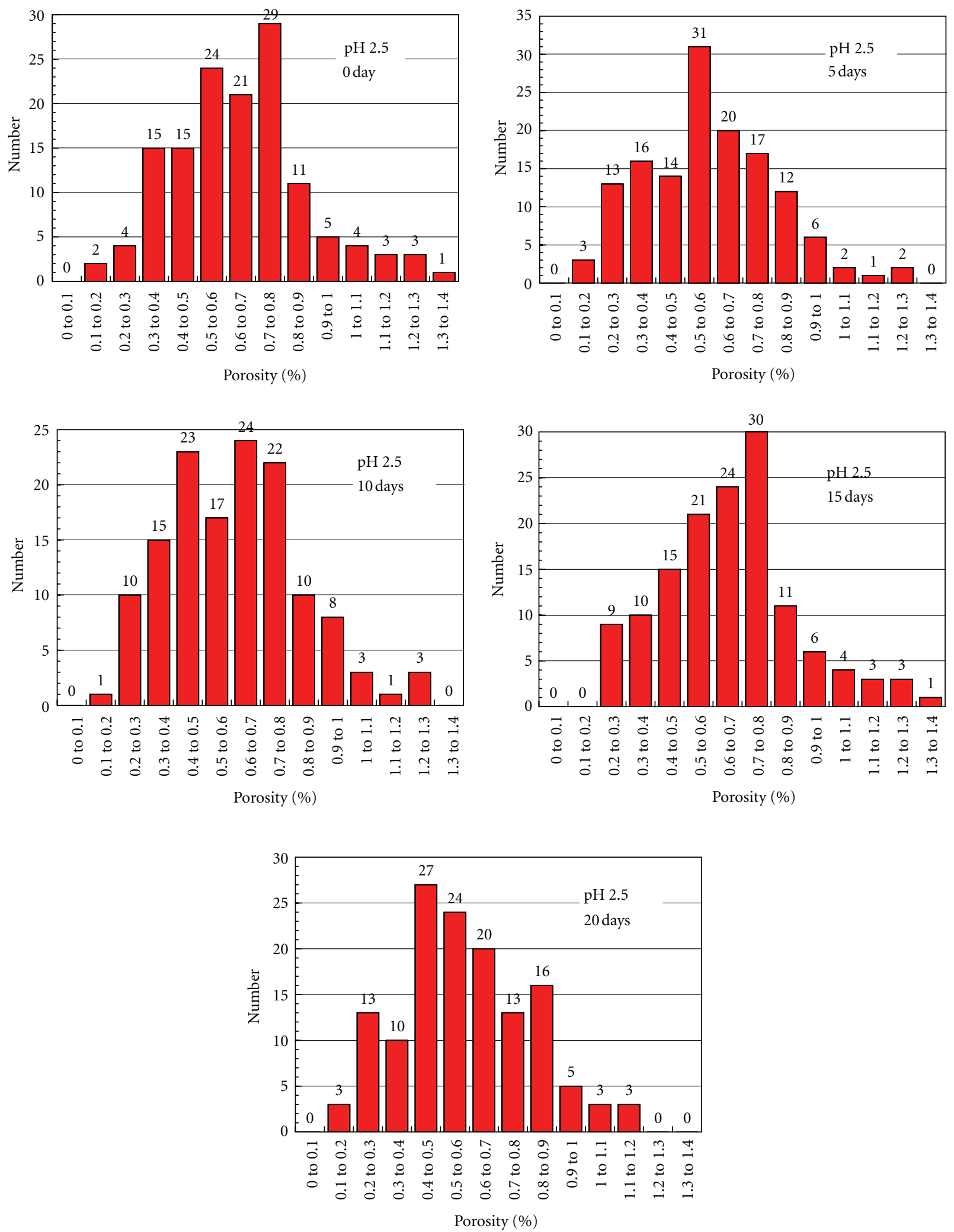

(b)

FIGURE 10: Probability distribution of the porosity inside concrete specimens. 


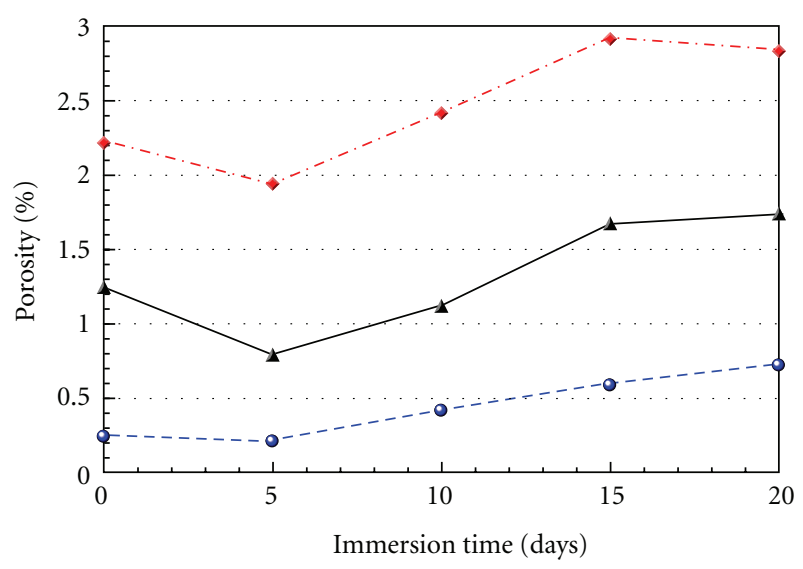

$\mathrm{pH} 1.5$

$\rightarrow-$ Maximum value

- ๑ - Minimum value

$\rightarrow$ Mean value

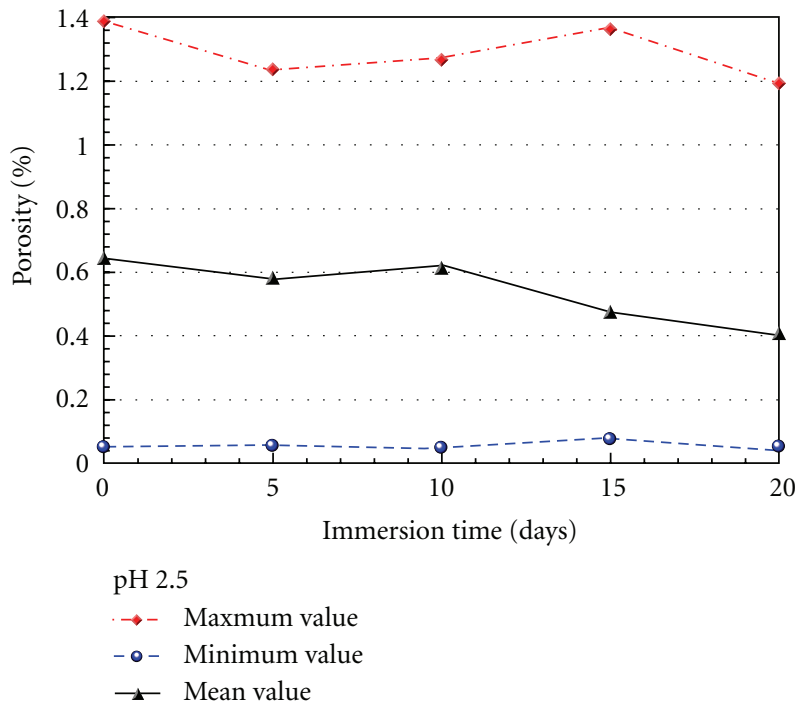

(b)

(a)

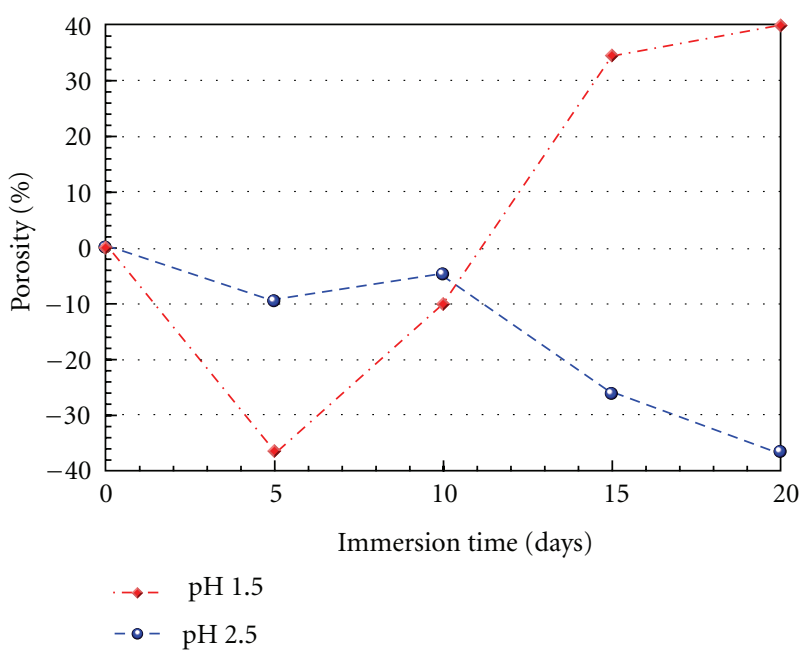

(c)

FIGURE 11: Relation between porosity ratio of concrete specimen and immersion time.

From Figure 9, it can be concluded that 79 is the optimal threshold value for the identification of the pore inside the concrete.

3.4.3. Development of Porosity Ratio inside Concrete. Using the above optimal threshold value, the digital images were processed by a programming calculation with Pro-Plus. The identification of pore inside the concrete was realized. Pore recognition graph of the concrete specimens under various damage states was then arrived at. The corresponding porosity ratios of $1202 \mathrm{D}$ section images for each concrete specimen are obtained, respectively. Based on the theory of probability statistics, the probabilistic characteristic of porosity distribution inside the concrete specimen come out. The analysis results of the porosity characteristics inside the concrete specimens under various damage states are shown in Figure 10, respectively.
From Figure 10, it is obvious that the distribution of the porosity ratio inside the concrete specimens obeys normal distribution. The maximum value, minimum, value and the average value of the concrete specimens are obtained. Development of average porosity ratio inside concrete specimen conditioned in the acidic solutions and immersion time is given in Figure 11.

It is shown that in the initial damage stage, the porosity ratio inside concrete exhibits a slight decrease. Higher acidity (lower $\mathrm{pH}$ value) of the solution results in higher increase. For example, the porosity ratio has increased 36.5 percent and 39.8 percent of the controlled specimen for specimens immersed in acid solutions with $\mathrm{pH}$ level of 1.5 for 5 and 20 days. While for those in the acid solutions with a $\mathrm{pH}$ level of 2.5 , the porosity ratio is always decreased in the first 20 days. Till 20 days, the porosity ratio has decreased 36.6 percent of the controlled specimen for 20 days. The changing 
trend shows a good agreement with the results provided by the mass analysis (shown in Figure 3).

\section{Conclusions}

This paper assesses the development of pore structure in concrete exposed to the acidic environment from multiscale levels. A series of tests, including physical, CT, SEM/EDS tests were performed on the concrete specimen in different prepared states. Weight loss, voids property, meso- and microstructure in the concrete specimens under various damage states are examined quantitatively. The results obtained on different scales are compared and discussed in detail. The conclusions from this study are summarized as follows.

(1) CT technology provides a useful tool to assess the porosity characteristic in the concrete. An optimal threshold value was suggested, and the pores inside the concrete are identified successfully. It can be drawn that the distribution of the porosity ratio in the concrete specimen sections obeys normal distribution.

(2) At the initial stage, the porosity ratio of deteriorated specimens increases slightly. Higher acidity of the solution leads to higher increase. The porosity ratio has increased 36.5 percent and 39.8 percent of the controlled specimen for specimens immersed in acid solutions with $\mathrm{pH}$ level of 1.5 for 5 and 20 days. While for those in the acid solutions with $\mathrm{pH}$ level of 2.5, the porosity ratio is always decreased till conditioning in the solutions for 20 days.

(3) The results obtained from macro-, meso- and microtests are in good correlation with each other.

\section{Acknowledgment}

This research was financially supported by the National Natural Science Foundation of China (Grants no. 51178069 and no. 50708010), Liaoning Provincial Funded project (Grant no. 20092149), and the Fundamental Research Funds for the Central Universities.

\section{References}

[1] D. Liu and D. Windslow, Pore Structure of Concrete. Joint Highway Research Project, Indiana Department of Transportation and Purdue University, West Lafayette, Ind, USA, 1986.

[2] A. B. Abell, K. L. Willis, and D. A. Lange, "Mercury intrusion porosimetry and image analysis of cement-based materials," Journal of Colloid and Interface Science, vol. 211, no. 1, pp. 3944, 1999.

[3] R. Kumar and B. Bhattacharjee, "Study on some factors affecting the results in the use of MIP method in concrete research," Cement and Concrete Research, vol. 33, no. 3, pp. 417-424, 2003.

[4] N. Hearn and R. D. Hooton, "Sample mass and dimension effects on mercury intrusion porosimetry results," Cement and Concrete Research, vol. 22, no. 5, pp. 970-980, 1992.
[5] A. Benouis and A. Grini, "Estimation of concrete's porosity by ultrasounds," Physics Procedia, vol. 21, pp. 53-58, 2011.

[6] Y. F. Fan, Z. Q. Hu, Y. Z. Zhang, and J. L. Liu, "Deterioration of compressive property of concrete under simulated acid rain environment," Construction and Building Materials, vol. 24, no. 10, pp. 1975-1983, 2010.

[7] F. Rendell, R. Jauberthie, and M. Grantham, Deteriorated Concrete, Inspection and Physicochemical Analysis, Thomas Telford Publishing, London, UK, 2002.

[8] P. W. Brown and J. R. Clifton, "Mechanisms of deterioration in cement-based materials and in lime mortar," Durability of Building Materials, vol. 5, no. 3, pp. 409-420, 1988.

[9] J. March and J. P. Skalny, Materials Science of Concrete: Sulfate Attack Mechanism, The American Ceramic Society, 1999.

[10] P. K. Metha and P. J. M. Monteiro, Concrete: Microstructure, Properties, and Materials, McGraw-Hill, New York, NY, USA, 2006. 

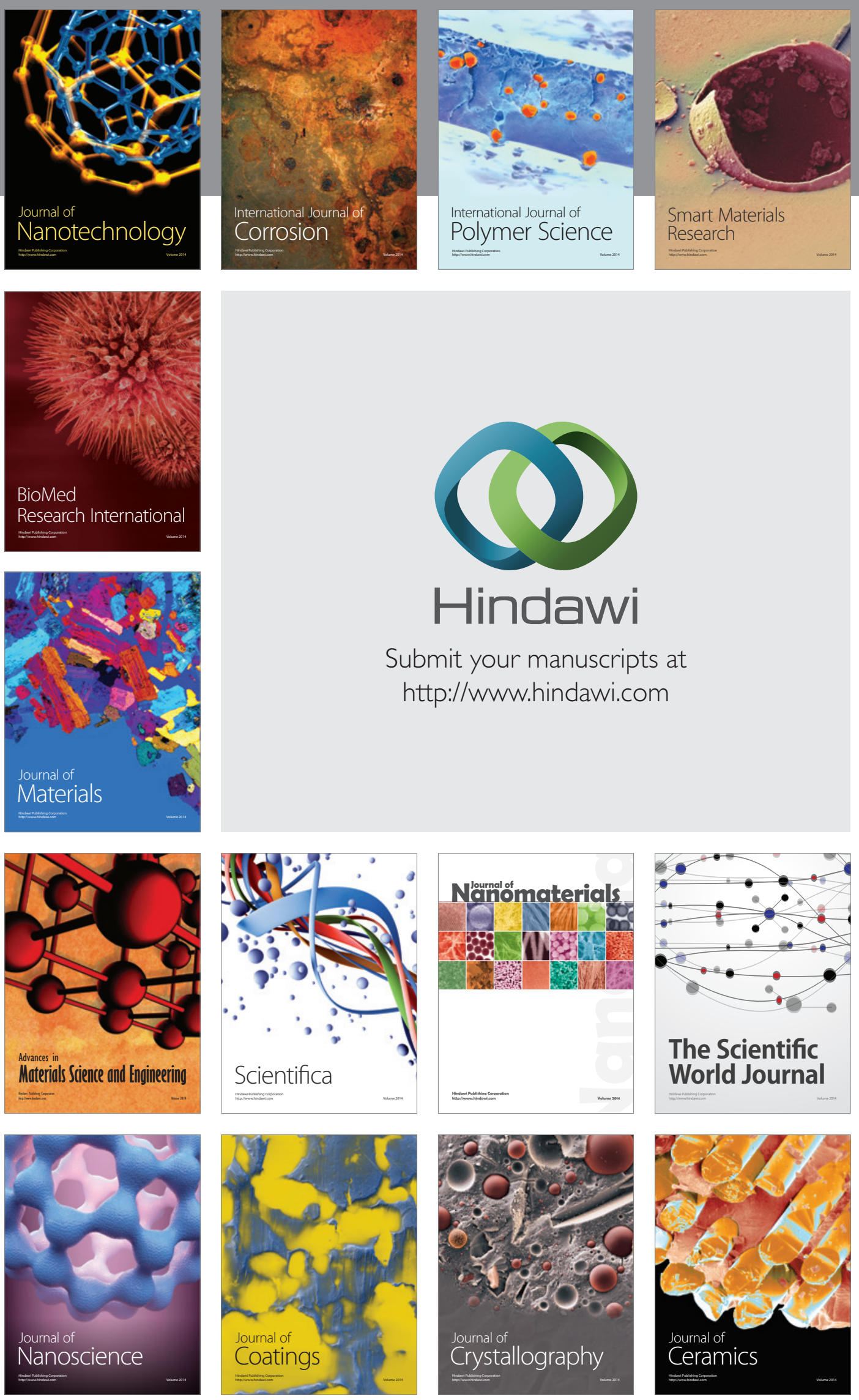

The Scientific World Journal

Submit your manuscripts at

http://www.hindawi.com

\section{World Journal}

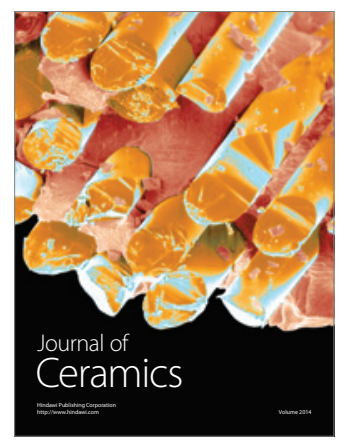

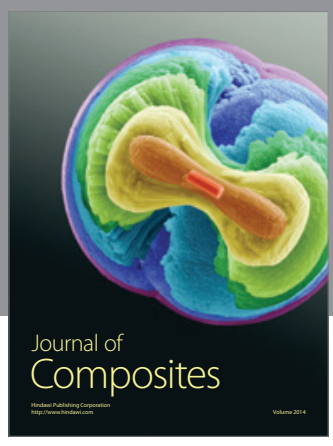
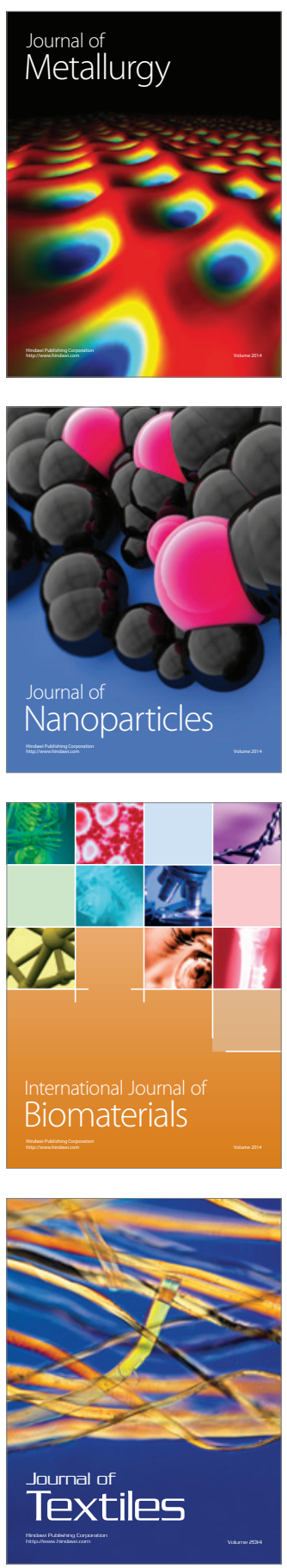